\title{
Analysis of $\mu$-Czochralski Technique Using Two-Dimensional Crystallization Simulator
}

\author{
Kuniaki Matsuki ${ }^{1}$, Ryusuke Saito ${ }^{1}$, Shuji Tsukamoto ${ }^{1}$, Mutsumi Kimura ${ }^{1,2}$, Ryoichi Ishihara ${ }^{3}$ \\ ${ }^{1}$ Department of Electronics and Informatics, Ryukoku University, Otsu, Japan; ${ }^{2}$ Joint Research Center for Science and Technology, \\ Ryukoku University, Otsu, Japan; ${ }^{3}$ Delft Institute of Microelectronics and Submicrontechnology, Delft University of Technology, \\ Delft, Netherlands. \\ Email: mutsu@rins.ryukoku.ac.jp
}

Received December $4^{\text {th }}, 2011$; revised January $5^{\text {th }}, 2012$; accepted January $16^{\text {th }}, 2012$

\begin{abstract}
$\mu$-Czochralski technique has been analyzed using two-dimensional crystallization simulator. It is observed that the temperature is relatively uniform in the entire $\mathrm{Si}$ region after the laser irradiation because the heat conductivity of the $\mathrm{Si}$ region is much higher than that of the underneath $\mathrm{SiO}_{2}$. Grain growth advances from the grain filter to the channel region and continues until it collides with what advances from random nucleation in the channel region. When the initial temperature is high, the random nucleation rarely occurs even under the supercooling condition, and the grain size becomes large. Moreover, it is qualitatively reproduced that the grain size increases as the irradiated energy of the laser irradiation increases.
\end{abstract}

Keywords: $\mu$-Czochralski Technique; Two Dimensional Crystallization Simulator; Grain Growth; Grain Filter; Grain Size

\section{Introduction}

$\mu$-Czochralski technique is a crystallization technique to enlarge poly-Si grains in thin-film transistors (TFTs) not only for flat panel displays and but also for general electronics $[1,2]$. In the $\mu$-Czochralski technique, grain filters are bored in underneath $\mathrm{SiO}_{2}$ films, amorphous-Si films are deposited and filled into the grain filters, excimer laser are irradiated to the amorphous-Si films, and grain growth advances from the grain filter and to the channel regions. Although the $\mu$-Czochralski technique has been experimentally analyzed in detail [3-6], the crystallization process should be theoretically clarified.

Recently, a two-dimensional (2-D) crystallization simulator has been developed and proposed as a practical evaluation tool for poly-Si TFTs [7]. In the 2-D crystallization simulator, random nucleation, crystal growth velocity, latent heat emission, and partial crystallization are modeled.

In this paper, we analyze the $\mu$-Czochralski technique using the 2-D crystallization simulator. We evaluate the temperature, grain growth, nucleation, etc. We try to reproduce the dependence of the grain size on the irradiated energy of the laser irradiation.

\section{2-D Crystallization Simulator}

The 2-D crystallization simulator is minutely explained in a previous paper [7]. Roughly speaking, the nucleation rate is defined as a function of temperature following a classical nucleation theory, and the crystal growth velocity is also defined as a function of temperature following a classical crystal growth theory. The partial crystallization model is used allowing the co-existence of the liquid and crystal phases even in a finite element. The simulation algorithm is composed of the phase transition and heat transfer algorithms. The thermal properties of $\mathrm{Si}$ and $\mathrm{SiO}_{2}$ are listed in the previous paper.

The grain filters are located in underneath $\mathrm{SiO}_{2}$ films, and grain seeds are put at the bottoms of the grain filters. The depth and diameter of the grain filters are $250 \mathrm{~nm}$ and $50 \mathrm{~nm}$. The grain seeds consist of fine grains formed during explosive crystallization at the beginning of the laser irradiation [3]. The channel regions are melt to the liquid$\mathrm{Si}$, and initial temperature of the liquid-Si is varied. The thickness of the channel regions is $250 \mathrm{~nm}$. The nucleation, grain growth, and temperature are successively calculated with the time after the laser irradiation. The calculation area is more than $2.5 \mu \mathrm{m}$. It should be noted that the initial temperature is set instead of giving the irradiated energy of the laser irradiation. The initial temperature is not the temperature at the laser irradiation, but the temperature at the start of the crystallization simulation.

\section{Simulation Results}

The distribution of the temperature in the grain filter, 
channel region, and underneath $\mathrm{SiO}_{2}$ is shown in Figure 1. Here, the initial temperature is $1800 \mathrm{~K}$, and the time after the laser irradiation is $80 \mathrm{~ns}$. It is observed that the temperature is relatively uniform in the entire Si region. This is because the heat conductivity of the $\mathrm{Si}$ region, 0.25 $\mathrm{WK}^{-1} \cdot \mathrm{cm}^{-1}$, is much higher than that of the underneath $\mathrm{SiO}_{2}, 0.014 \mathrm{WK}^{-1} \cdot \mathrm{cm}^{-1}$. As seen in Figure 1, the temperature at the top of the grain filter is slightly high owing to the latent heat released during the grain growth from the grain filter. Moreover, the temperature at the right side in the calculation area is also slightly high owing to the latent heat released during the grain growth from the random nucleation, as seen in Figure 2(b).

The advance of the grain growth in the $\mu$-Czochralski process is shown in Figure 2. Here, the initial temperature is $1800 \mathrm{~K}$, and the time after the laser irradiation is 40 $200 \mathrm{~ns}$. It is observed that the grain growth advances from the grain filter to the channel region. The random nucleation occurs, as seen in Figures 2(b) and (c), and the grain growth continues until it collides with what advances from random nucleation in the channel region, as seen in Figures 2(c) and (d). As a result, the center grain from the grain filter is surrounded by the many grains from random nucleation, as seen in Figure 2(d).

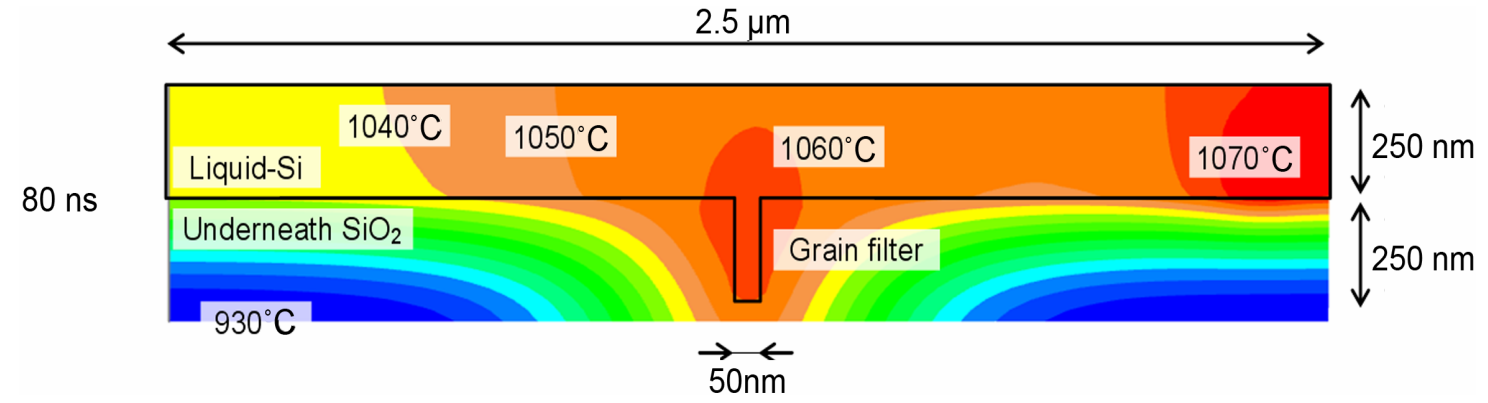

Figure 1. Distribution of the temperature in the grain filter, channel region, and underneath $\mathrm{SiO}_{2}$.

(a) $40 \mathrm{~ns}$

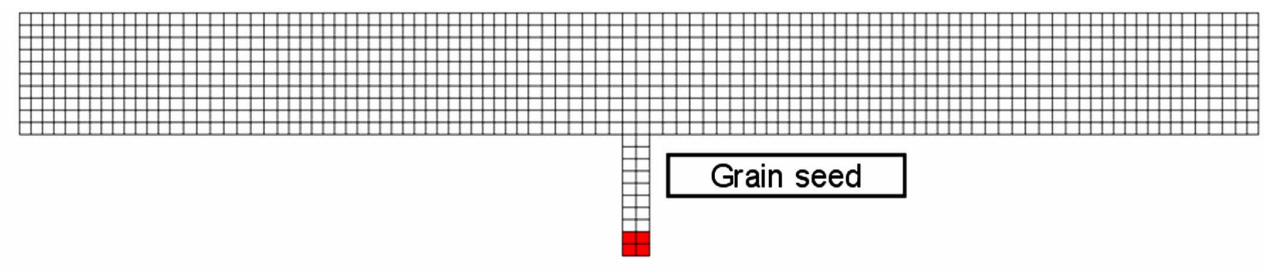

(b) $80 \mathrm{~ns}$

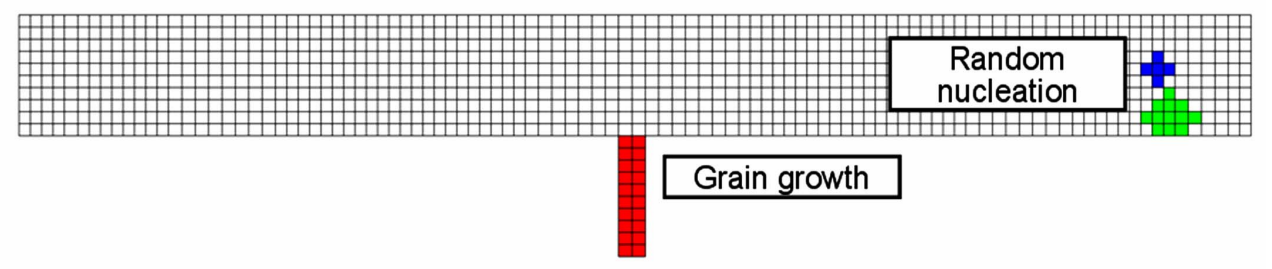

(c) $120 \mathrm{~ns}$

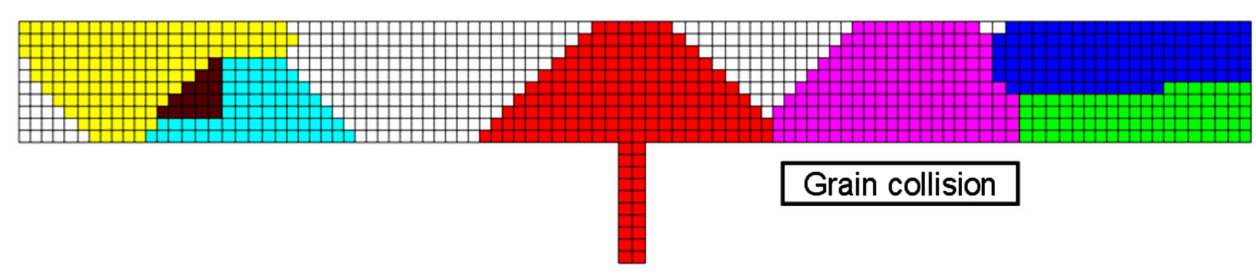

(d) $200 \mathrm{~ns}$

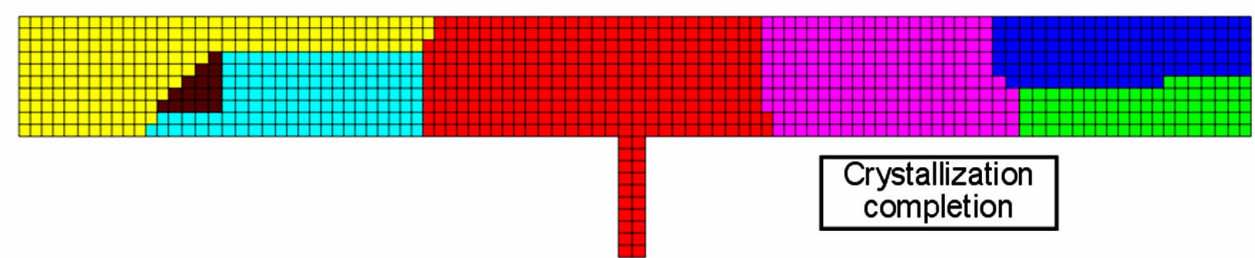

Figure 2. Advance of the grain growth in the $\mu$-Czochralski process. 
The dependence of the grain growth on the initial temperature is shown in Figure 3. Here, the initial temperature is varied from $1700 \mathrm{~K}$ to $2200 \mathrm{~K}$. The grain size is small for the initial temperatures of $1700 \mathrm{~K}$ and $1800 \mathrm{~K}$, as seen in Figures 3(a) and (b). This is because the random nucleation occurs in the channel region during the grain growth from the grain filter. On the other hand, the grain size increases for the initial temperature more than $1900 \mathrm{~K}$ as the initial temperature increases, as seen in Figures 3(c)-(e). This is because the grain growth advances from the grain filter while the temperature in the channel region is high and the Si stays liquid-Si. Since the nucleation rate is small considering that the time scales of the laser irradiation and following cooling process are short, the random nucleation rarely occurs even under the supercooling condition, and the grain size becomes large.

The dependence of the grain size on the initial temperature is shown in Figure 4. Here, the initial temperature is varied from $1700 \mathrm{~K}$ to $2400 \mathrm{~K}$. It is qualitatively reproduced that the grain size increases as the initial temperature increases, which corresponds to the irradiated energy of the laser irradiation. This result is consistent with experimental results [8].

\section{Conclusions}

$\mu$-Czochralski technique has been analyzed using 2-D crystallization simulator. It was observed that the temperature is relatively uniform in the entire liquid-Si after the laser irradiation because the heat conductivity of the $\mathrm{Si}$ region is much higher than that of the underneath $\mathrm{SiO}_{2}$. Grain growth advances from the grain filter to the channel region and continues until it collides with what advances from random nucleation in the channel region. When the initial temperature is high, the random nucleation rarely occurs even under the supercooling condition, and the grain size becomes large. Moreover, it was qualitatively reproduced that the grain size increases as the irradiated energy of the laser irradiation increases.

It is obvious that the lateral grain growth such as $\mu-C z o-$ chralski technique cannot be reproduced using one-dimensional crystallization simulator, whereas it is expected that

(a) $1700 \mathrm{~K}$

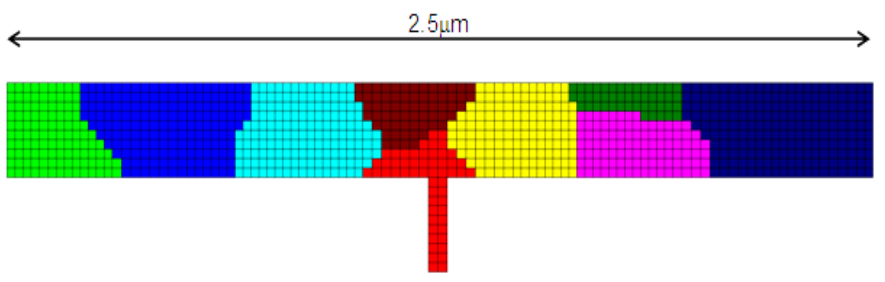

(b) $1800 \mathrm{~K}$

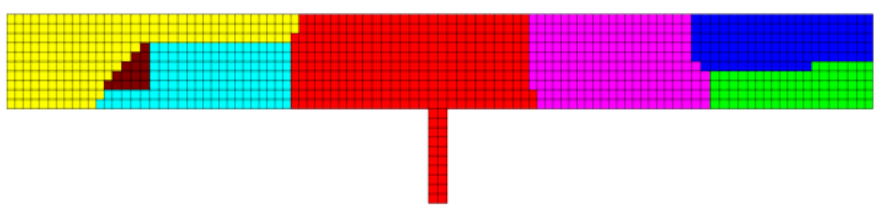

(c) $1900 \mathrm{~K}$

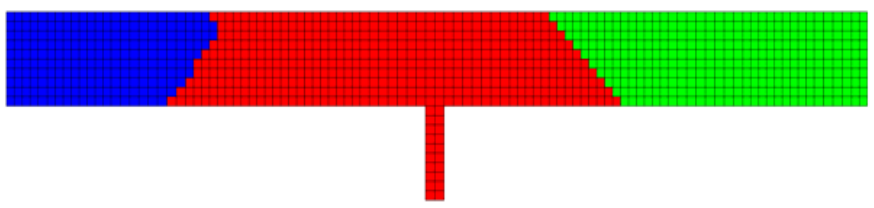

(d) $2000 \mathrm{~K}$
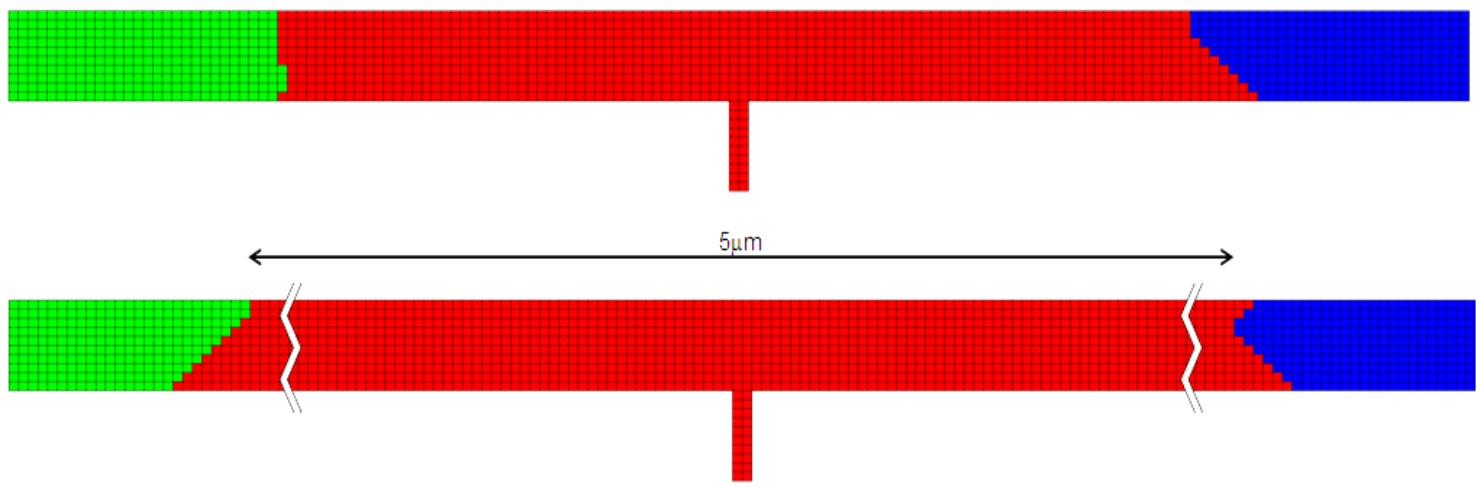

Figure 3. Dependence of the grain growth on the initial temperature. 


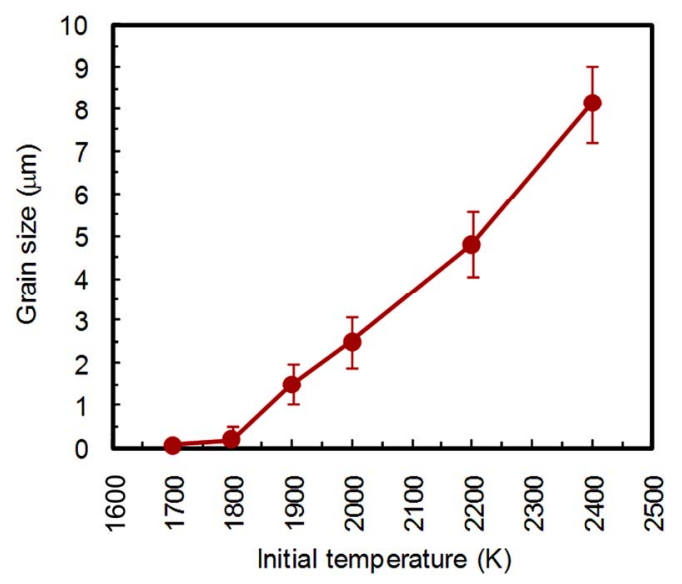

Figure 4. Dependence of the grain size on the initial temperature.

it can be reproduced using three-dimensional simulator, which consumes terribly long computation time. It is meaningful that the lateral grain growth can be at least qualitatively reproduced using two-dimensional crystallization, which consumes acceptable computation time, although a certain cross section is only considered.

\section{REFERENCES}

[1] P. C. van der Wilt, B. D. van Dijk, G. J. Bertens, R. Ishihara and C. I. M. Beenakker, "Formation of LocationControlled Crystalline Islands Using Substrate-Embedded Seeds in Excimer-Laser Crystallization of Silicon Films," Applied Physics Letters, Vol. 79, No. 12, 2001, pp. 18191821. doi:10.1063/1.1402641

[2] N. Saputra, M. Danesh, A. Baiano, R. Ishihara, J. R. Long, N. Karaki and S Inoue, "An Assessment of $\mu$-Czo-chralski, Single-Grain Silicon Thin-Film Transistor Technology for Large-Area, Sensor and 3-D Electronic Integra- tion," IEEE Journal of Solid-State Circuits, Vol. 43, No. 7, 2008, pp. 1563-1576. doi:10.1109/JSSC.2008.922404

[3] R. Ishihara, , P. C. van der Wilt, B. D. van Dijk, A. Burtsev, J. W. Metselaar and C. I. M. Beenakker, "Advanced Excimer-Laser Crystallization Process for Single-Crystalline Thin Film Transistors," Thin Solid Films, Vol. 427, No. 1-2, 2003, pp. 77-85. doi:10.1016/S0040-6090(02)01250-6

[4] R. Ishihara, Y. Hiroshima, D. Abe, B. D. van Dijk, P. C. van der Wilt, S. Higashi, S. Inoue, T. Shimoda, J. W. Metselaar and C. I. M. Beenakker, "Single-Grain Si TFTs with ECR-PECVD Gate $\mathrm{SiO}_{2}$," IEEE Transactions on Electron Devices, Vol. 51, No. 3, 2004, pp. 500-502. doi:10.1109/TED.2004.823326

[5] V. Rana, R. Ishihara, Y. Hiroshima, D. Abe, S. Inoue, T. Shimoda, W. Metselaar and K. Beenakker, "Dependence of Single-Crystalline Si TFT Characteristics on the Channel Position inside a Location-Controlled Grain," IEEE Transactions on Electron Devices, Vol. 52, No. 12, 2005, pp. 2622-2628. doi:10.1109/TED.2005.859689

[6] R. Ishihara, D. Danciu, F. Tichelaar, M. He, Y. Hiroshima, S. Inoue, T. Shimoda, J.W. Metselaar and C. I. M. Beenakker, "Microstructure Characterization of LocationControlled Si-Islands Crystallized by Excimer Laser in the $\mu$-Czochralski (Grain Filter) Process," Journal of Crystal Growth, Vol. 299, No. 2, 2007, pp. 316-321. doi:10.1016/i.jcrysgro.2006.12.010

[7] K. Matsuki, R. Saito, S. Tsukamoto and M. Kimura, "Two-Dimensional Simulator of Laser Crystallization for Polycrystalline-Silicon Thin-Film Transistors," IEEE Transactions on Semiconductor Manufacturing, Vol. 24, No. 3, 2011, pp. 472-476. doi:10.1109/TSM.2011.2154368

[8] R. Vikas, R. Ishihara, Y. Hiroshima, D. Abe, S. Inoue, T. Shimoda, W. Metselaar and K. Beenakker, "Capping Layer on Thin Si Film for $\mu$-Czochralski Process with Excimer Laser Crystallization," Japanese Journal of Applied Physics, Vol. 45, No. 5B, 2006, pp. 4340-4343. doi:10.1143/JJAP.45.4340 